UB-ECM-PF 94/11

April, 1994

\title{
Asymptotic regimes in quantum gravity at large distances and running Newtonian and cosmological constants
}

\author{
E. Elizalde 日 \\ Center of Advanced Studies, CSIC, Camí de Sta. Bàrbara, 17300 Blanes, \\ and Department E.C.M., Faculty of Physics, University of Barcelona, \\ Diagonal 647, 08028 Barcelona, Catalonia, Spain \\ S.D. Odintsov 9 \\ Department E.C.M., Faculty of Physics, University of Barcelona, \\ Diagonal 647, 08028 Barcelona, Catalonia, Spain \\ and Tomsk Pedagogical Institute, 634041 Tomsk, Russia \\ and

\section{I.L. Shapiro} \\ Tomsk Pedagogical Institute, 634041 Tomsk, Russia \\ and Department of Physics, Hiroshima University, Higashi-Hiroshima 724, Japan
}

\begin{abstract}
We consider a multiplicatively renormalizable higher-derivative scalar theory which is used as an effective theory for quantum gravity at large distances (infrared phase of quantum gravity). The asymptotic regimes (in particular, the asymptotically free infrared regime) for the coupling constants - specifically the Newtonian and the cosmological constant - are obtained. The running of the Newton and cosmological constants in the infrared asymptotically free regime may be relevant for solving the cosmological constant problem and for estimating the leading-log corrections to the static gravitational potential.
\end{abstract}

\footnotetext{
${ }^{1}$ E-mail: eli@zeta.ecm.ub.es, eli@ebubecm1.bitnet

${ }^{2}$ E-mail: odintsov@ebubecm1.bitnet

${ }^{3}$ E-mail: shapiro@fusion.sci.hiroshima-u.ac.jp
} 
1. Introduction. There is a longstanding hope that the cosmological constant problem (for a review see [1]) may be solved with the help of quantum gravity (QG) which, on the other hand, does not yet exist as a consistent theory. Recently a very interesting approach to solve the cosmological constant problem based on an effective theory of the conformal factor has been suggested in Ref. [2] (see, also [3]). The effective theory for the conformal factor, obtained as a result of integrating over the conformal anomaly [4] (the derivation of the anomaly induced action is done in [7,8]), has been used to describe QG at large distances (larger then the horizon scale) in the far infrared (IR) [2]. (For a different approach to QG in the far infrared, see [5]). The anomalous scaling dimension for the conformal factor has been derived in the IR stable fixed point, what gives a way to fix the cosmological constant. Note that the main idea of [2] is very similar to the standard approach to the Polyakov action in two dimensions.

In the present letter we discuss the effective theory of the conformal factor aiming at the description of QG at large distances from a different viewpoint in comparison with [2]. We shall not consider this theory exactly in the IR stable fixed point. Instead, we shall write the renormalization group (RG) equations (corresponding to the more general model of [6]) for all the effective couplings of the theory. The special asymptotically free solutions for the effective couplings generalize the effective theory of the conformal factor of Ref. [2]. We find a general solution which has two different asymptotical regimes consistent in the IR (large distances) or in the UV limit. These RG solutions are used to discuss the running of the effective Newtonian and cosmological constants. Some possible applications to the case of a static gravitational potential are pointed out.

2. Solutions of the RG equations for the general model. Let us start from the model of Ref. [6] which is renormalizable in a generalized sense. The classical action has the form

$$
\begin{gathered}
S=\int d^{4} x \sqrt{-g}\left\{b_{1}(\varphi)(\square \varphi)^{2}+b_{2}(\varphi)\left(\nabla_{\mu} \varphi\right)\left(\nabla^{\mu} \varphi\right) \square \varphi+b_{3}(\varphi)\left[\left(\nabla_{\mu} \varphi\right)\left(\nabla^{\mu} \varphi\right)\right]^{2}+\right. \\
+b_{4}(\varphi)\left(\nabla_{\mu} \varphi\right)\left(\nabla^{\mu} \varphi\right)+b_{5}(\varphi)+c_{1}(\varphi) R\left(\nabla_{\mu} \varphi\right)\left(\nabla^{\mu} \varphi\right)+c_{2}(\varphi) R^{\mu \nu}\left(\nabla_{\mu} \varphi\right)\left(\nabla_{\nu} \varphi\right) \\
\left.+c_{3}(\varphi) R \square \varphi+a_{1}(\varphi) R_{\mu \nu \alpha \beta}^{2}+a_{2}(\varphi) R_{\mu \nu}^{2}+a_{3}(\varphi) R^{2}+a_{4}(\varphi) R\right\} .
\end{gathered}
$$

Note that $[\varphi]=0$ and the only $\varphi$ is a quantum field, that is, the metric is purely classical. We suppose that $c_{1,2}, a_{1,2,3,4}, b_{1,2,3}$ are constants and that $c_{3}(\varphi)=c_{31} \varphi+c_{32}$. Then this theory is multiplicatively renormalizable in the usual sense (for the above choice of $\left.b_{4}(\varphi), b_{5}(\varphi)\right)$. The renormalization group equations for all the effective couplings (supposing the standard renormalization of the scalar $\varphi$ ) have been derived in [6]

$$
\frac{d b_{2}(t)}{d t}=-10 b_{2}(t) b_{3}(t)+\frac{15}{4} b_{2}^{3}(t), b_{2}(0)=b_{2},
$$




$$
\begin{gathered}
\frac{d b_{3}(t)}{d t}=-10 b_{3}^{2}(t)+5 b_{2}^{2}(t) b_{3}(t), b_{3}(0)=b_{3}, \\
\frac{d c_{31}(t)}{d t}=\frac{5}{2} b_{2}^{2}(t) c_{31}(t), c_{31}(0)=c_{31} . \\
\frac{d c_{1}(t)}{d t}=\frac{2}{3} b_{3}(t)\left[9 c_{31}(t)-9 c_{1}(t)-2 c_{2}(t)+2\right]+\frac{5}{2} b_{2}^{2}(t) c_{1}(t), c_{1}(0)=c_{1} \\
\frac{d c_{2}(t)}{d t}=-\frac{1}{3}\left[2 c_{2}(t) b_{3}(t)-b_{2}^{2}(t)+4 b_{3}(t)\right]+\frac{5}{2} b_{2}^{2}(t) c_{2}(t), c_{2}(0)=c_{2}, \\
\frac{d c_{32}(t)}{d t}=\frac{1}{3} b_{2}(t)\left[-9 c_{1}(t)-2 c_{2}(t)+2\right]+\frac{5}{4} b_{2}^{2}(t) c_{32}(t), c_{32}(0)=c_{32} .
\end{gathered}
$$

Finally, the RG equations for the effective vacuum couplings are

$$
\begin{gathered}
\frac{d a_{1}(t)}{d t}=0, a_{1}(0)=a_{1}, \\
\frac{d a_{2}(t)}{d t}=-\frac{1}{12} c_{2}^{2}(t)-2 c_{2}(t)-\frac{1}{15}, a_{2}(0)=a_{2} \\
\frac{d a_{3}(t)}{d t}=\frac{-3\left[4 c_{1}(t)+c_{2}(t)-4 c_{31}(t)\right] o 2+c_{2}^{2}(t)}{48}-\frac{2 c_{31}(t)-2 c_{1}(t)-c_{2}(t)}{6}-\frac{1}{30}, a_{3}(0)=a_{3}, \\
\frac{d a_{4}(t)}{d t}=\frac{1}{2} b_{4}(t)\left[4 c_{31}(t)-4 c_{1}(t)-c_{2}(t)+\frac{2}{3}\right], a_{4}(0)=a_{4} .
\end{gathered}
$$

Below we consider the general and special asymptotic regimes for the renormalization group equations (2) and then concentrate our attention on the following particular choice of functions $b_{4}(\varphi)$ and $b_{5}(\varphi)$

$$
b_{4}(\varphi)=\gamma \exp (2 \alpha \varphi), b_{5}(\varphi)=\lambda \exp (4 \alpha \varphi)
$$

which corresponds to the model of Ref. [2].

The general solution of equations (2) possesses some very interesting features. For the sake of simplicity we introduce new variables $x, y, z$ according to $4 z=b_{2}^{2}, y=b_{3}, x=z / y$. Then one can easily obtain the equation

$$
\frac{d y}{y}=\frac{(2 x-1) d x}{x^{2}-x}
$$

and, moreover, three special solutions for (2):

$$
\begin{aligned}
& z=y, \quad \text { that is } b_{2}^{2}=4 b_{3}, \\
& z=0, \quad \text { that is } b_{2}^{2}=0, \\
& y=0, \quad \text { that is } b_{3}=0 .
\end{aligned}
$$

Let us now explore (7). This equation leads to the following relation between $y$ and $z$

$$
z=y\left[\frac{1}{2} \pm \sqrt{\frac{y}{c_{1}}+\frac{1}{4}}\right] .
$$


Substituting (9) into the first Eq. of (2), we get

$$
\frac{d y}{y^{2} \sqrt{1+c y}}= \pm 10 d t, \quad c=\frac{1}{4 c_{1}} \text {. }
$$

Integrating (10), we finally obtain

$$
\pm 10 t+c_{2}=\frac{1}{2} \ln \left|\frac{v-1}{v+1}\right|-\frac{1}{2} \frac{v}{v^{2}-1}, \quad v^{2}=1+c y .
$$

Eq. (9) can be viewed as a first integral of the system (2), in fact it can be rewriten in the form:

$$
b_{2}^{4}-4 b_{2}^{2} b_{3}=k_{1} b_{3}^{3},
$$

which is a family of curves on the plane $b_{2}, b_{3}$ with parameter $k_{1}$ (constant of motion) to be determined from the initial values $b_{2}(0)$ and $b_{3}(0)$. Another first integral can be easily obtained by reducing the system (2) to an exact diferential equation by means of the integrating factor $b_{2}^{9} b_{3}^{8}$. We then get

$$
12 b_{2}^{10} b_{3}^{10}-5 b_{2}^{12} b_{3}^{9}=k_{2}
$$

Again, the parameter $k_{2}$ (a second constant of motion) is to be fixed from the initial values $b_{2}(0)$ and $b_{3}(0)$. It can be seen immediately that, in general, the two families of curves do not intersect (whenever they do they yield a trivial, constant trajectory). From the last expression we get the following particular solution: if $b_{3}(0)=(5 / 12) b_{2}(0)^{2}$ then, for any $t, b_{3}(t)=(5 / 12) b_{2}(t)^{2}$ and solving for the dynamics (2) one obtains

$$
b_{2}(t)=\frac{b_{2}}{\sqrt{1+\frac{5}{6} b_{2}^{2} t}}, \quad b_{3}(t)=\frac{5 b_{2}^{2}}{2\left(6+5 b_{2}^{2} t\right)} .
$$

We observe that (9) and (10) admit two possible asymptotic regimes for $y$ and $z$. In both regimes $y$ tends to zero when $t \rightarrow \infty$. The first regime corresponds to the positive sign in (9). Then, it is easy to see that in the $t \rightarrow-\infty$ limit (large distance) the couplings have infinitesimal difference with the special solution (8a), where we have

$$
b_{2}^{2}(t)=\frac{2 b_{2}^{2}}{2-5 b_{2}^{2} t}, \quad b_{3}(t)=\frac{b_{2}^{2}}{2\left(2-5 b_{2}^{2} t\right)} .
$$

At the same time this regime leads to the zero-charge problem in the UV limit $t \rightarrow+\infty$.

The second regime corresponds to the negative sign in (9). After tiny algebra we obtain that in this case $z \sim y^{2}$ and moreover, from (10) we get that $y(t) \sim t^{-1}$ in both limits. So we see that this regime is in some sense similar to the special solution (8b). Substituting $b_{2}^{2} \ll b_{3}$ into (2), we get the following regime, which can be asymptotically free in both the UV and IR limits [6]

$$
b_{2}(t) \simeq \frac{b_{2}}{1+10 b_{3} t}, \quad b_{3}(t) \simeq \frac{b_{3}}{1+10 b_{3} t} .
$$


Here, we have asymptotic freedom in the UV or IR limits, and the result depends on the sign of $b_{3}$. It is very interesting to note that the sign of $b_{3}$ is not connected with any physical quantity (contrary to what happens in the $\lambda \varphi^{4}$ theory where the wrong sign of $\lambda$ corresponds to an unstable classical potential). Hence one can choose any sign of $b_{3}$ and have asymptotic freedom in the IR or in the UV region. (Notice, however, that in the model [2] $b_{3}$ is always negative.) Therefore, the theory under discussion posesses some unusual property in this point. Observe also that from the discussion above it follows that one can easily find the running couplings corresponding to the rest of the parameters of the theory.

3. Running couplings in QG at large distances. Now we shall study the running of the coupling constants in the effective theory of the conformal factor. This theory has been discussed in Ref. [2], but only in the fixed point $b_{2}=b_{3}=0$. On the countrary, we shall here consider all the possible asymptotic regimes for these couplings. Notice that the correspondence between our model and the model of [2] is obtained by setting

$$
b_{1}=-\frac{Q^{2}}{(4 \pi)^{2}}, \quad b_{2}=-2 \zeta \alpha, \quad b_{3}=-\zeta^{2} \alpha, \quad b_{4}=\gamma \exp (2 \alpha \varphi), \quad b_{5}=-\frac{\lambda}{\alpha^{2}} \exp (4 \alpha \varphi)
$$

where $Q^{2}$ is the four-dimentional central charge, $\alpha$ the scaling dimension for $\varphi, \gamma$ the gravitational coupling, and $\lambda$ is the cosmological constant. Further, we will use the notations (1). As one can see, the theory under consideration differs from the general model (1) by the choice (6) for the couplings. Such a theory can be renormalized in two different ways. One can consider $b_{1}, b_{2}, b_{3}$ as arbitrary constants, which have to be renormalized as well as $a_{1}, a_{2}, a_{3}$ and $c_{1}, c_{2}, c_{3}$. Or one can either consider $b_{1}$ as a nonessential constant and renormalize $\alpha$ instead of $b_{1}$. Here we will adopt the second point of view. The $\mathrm{RG}$ equations for $b_{2}(t), b_{3}(t), c_{1}(t), c_{2}(t), c_{3}(t), a_{1}(t), a_{2}(t), a_{3}(t)$ have the same form (2)-(5). At the same time, we easily find the RG equations for $\gamma(t), \alpha(t), \lambda(t)$, which are a particular case of $(3)$ :

$$
\begin{gathered}
\frac{d \alpha(t)}{d t}=\frac{5}{4} b_{2}^{2}(t), \alpha(0)=\alpha \\
\frac{d \gamma(t)}{d t}=\gamma(t)\left[b_{2}^{2}(t)+4 \alpha^{2}(t)+6 b_{2}(t) \alpha(t)\right], \quad \gamma(0)=\gamma \\
\frac{d \lambda(t)}{d t}=16 \alpha^{2}(t) \lambda(t)-\gamma^{2}(t), \quad \lambda(0)=\lambda .
\end{gathered}
$$

These equations can be solved indepently of (4) and (5), because the corresponding running couplings do not change when we restrict the theory to live in flat space. Next, since in all asymptotic regimes the basic couplings $b_{2}, b_{3}$ have just the same qualitative asymptotic behaviour, we can restrict ourselves by just one of them. Let us consider the 
special solution $(8 \mathrm{~b})$, and put $\frac{1}{4} b_{3}(t)=b_{2}^{2}(t)=b_{2}^{2}\left(1-\frac{5}{2} b_{2}^{2} t\right)^{-1}$. Then we easily find the solution of (13) in the form

$$
\begin{gathered}
\alpha(t)=\frac{\alpha}{\sqrt{1-\frac{5}{2} b_{2}^{2} t}} \\
\gamma(t)=\gamma\left(1-\frac{5}{2} b_{2}^{2} t\right)^{-\frac{2\left(b_{2}^{2}+4 \alpha^{2}+6 b_{2} \alpha\right)}{5 b_{2}^{2}}}, \\
\lambda(t)=\frac{2 \gamma}{3\left(b_{2}^{2}+8 \alpha^{2}-4 b_{2} \alpha\right)}\left(1-\frac{5}{2} b_{2}^{2} t\right)^{\frac{\left.3 b_{2}^{2}-8 \alpha^{2}-12 b_{2} \alpha\right)}{5 b_{2}^{2}}} \\
+\left[\lambda-\frac{2 \gamma}{3\left(b_{2}^{2}+8 \alpha^{2}-4 b_{2} \alpha\right)}\right]\left(1-\frac{5}{2} b_{2}^{2} t\right)^{-\frac{32 \alpha^{2}}{5 b_{2}^{2}}}
\end{gathered}
$$

That solution describes qualitatively the IR phase of quantum gravity in frames of our effective theory. Let us now give a brief analysis of the behaviour of the effective couplings $\lambda(t), \gamma(t)$. The asymptotics essentially depend on the value of the ratio $u=\alpha / b_{2}$. For example, if $-3-\sqrt{5}<u<-3+\sqrt{5}$, then $\lambda(t) \rightarrow 0$ and $\gamma(t) \rightarrow \infty$ in the IR limit. When $u=-3 \pm \sqrt{5}$, then $\lambda(t) \rightarrow 0$ and $\gamma(t)=\gamma=$ const.

Let us discuss the behaviour of the running Newton and cosmological constants in more detail. The standard expression for the Newtonian potential has the form

$$
V(r)=-\frac{G m_{1} m_{2}}{r}
$$

where in our notation $\gamma=3 /(8 \pi G)$. Observe that, in $R^{2}$-gravity, already at the classical level there appear corrections of Yukawa type to the classical gravitational potential (20) (see Ref. [9], and for a review of quantum $R^{2}$-gravity see [10]).

We are working in terms of an effective theory of quantum gravity. Such a description is valid in the far infrared (large distances) and the extremely small exponentially suppressing corrections of Ref. [9] to the potential (20) are not important in such case. However, the fact that the Newton coupling becomes the running coupling is very important for its possible cosmological applications. Indeed, we are guided by the well-known example of the electrostatic potential in quantum electrodynamics. Here, this potential can be alternatively obtained from the classical part of the potential by the change of electric charge to corresponding running coupling with $t=\left(1 / 32 \pi^{2}\right) \ln \left(r_{0}^{2} / r^{2}\right)$. Hence, the Wilsonian (or renormalization-group improved) electrostatic potential coincides with the explicit calculation of the electrostatic potential in perturbation theory.

In the same way, in Refs. [11] and [12] it was suggested to use the Wilsonian gravitational potential (20) (i.e., with running gravitational coupling constant) for a possible solution of the dark matter problem [12] and of the cosmological constant problem (in the wormhole context). However, in Ref. [12] the running Newton constant that was obtained 
from quantum $R^{2}$-gravity in the ultraviolet asymptotically free regime was used. Surely, this is not quite consistent for estimating the infrared effects.

Instead, we have used the running Newton coupling constant calculated in the effective theory, with the aim to describe quantum gravity in the far infrared (large distances). Then the leading quantum corrections for the Newtonian potential together with the classical part yields (see Eqs. (18) and (20)):

$$
\begin{gathered}
V(r)=-\frac{G_{0} m_{1} m_{2}}{r}\left(1-\frac{5 b_{2}^{2}}{64 \pi^{2}} \ln \frac{r_{0}^{2}}{r^{2}}\right)^{-\frac{2\left(b_{2}^{2}+4 \alpha^{2}+6 b_{2} \alpha\right)}{5 b_{2}^{2}}} \\
\simeq-\frac{G_{0} m_{1} m_{2}}{r}\left(1+\frac{2\left(b_{2}^{2}+4 \alpha^{2}+6 b_{2} \alpha\right)}{32 \pi^{2}} \ln \frac{r_{0}^{2}}{r^{2}}\right) .
\end{gathered}
$$

Here $G_{0}$ is the initial value of the Newton constant at distance $r_{0}$. Eq. (21) shows the leading-log corrections to the classical gravitational potential in the context of our effective theory of quantum gravity. For a rough estimation of the quantum correction in (21) we take $\alpha=1$ (classical value) and use $b_{2}=-2\left(2 \tilde{b}+2 \tilde{b}^{\prime}+3 \tilde{b}^{\prime \prime}\right)$, where the conformal anomaly $T=\tilde{b}\left(F+\frac{2}{3} \square R\right)+\tilde{b}^{\prime} G+\tilde{b}^{\prime \prime} \square R$ coefficients are known [4]. For instance, for scalars they are proportional to the effective number of matter fields. Hence, the quantum correction to the Newtonian gravitational potential is proportional to the square of the effective number of matter fields. Of course, this correction is far too small to be experimentally measured. However, this potential (21) might growe within the region $u<-3-\sqrt{5}$ or $u>-3+\sqrt{5}$, and this might lead to more reasonable implications for the solution of the dark matter problem than just use of the UF asymptotically free running couplings of quantum $R^{2}$-gravity. Let us recall that, recently, leading corrections to the Newtonian potential have been calculated in the one-loop approach for the Einstein theory considered as an effective theory of quantum gravity [13], where logarithmic corrections were not found. In our approach we get a definitely better approximation to the Newton potential, because we use a Wilsonian potential that sums all leading logarithms of the perturbation theory. This is, of course, a richer situation in comparison with what one gets when taking into account the one-loop corrections only.

Notice that the running cosmological constant (19) may quickly decay in the infrared region, as it follows from the explicit expression (see also [14]). This fact depends very much on the choice of the parameters of the theory and gives an indication of the possibility of solving the cosmological constant problem as a result of infrared effects in quantum gravity.

Concerning the behaviour of $\gamma(t)$ and $\lambda(t)$ on the general solutions of $(2)$, since the solutions (11) and (12) are related with each other by the obvious change of $t$, it is clear that the above picture will preserve its structure when the asymptotic regime (11) is 
used. That is why it is not necessary to study the details of the solutions for the couplings $c_{1,2,3}(t)$ and $a_{1,2,3}$. This analysis has been already performed in a previous paper, Ref. [6], in the framework of the asymptotic regime (12), and will be surely the same for the special solution (11), that is just the starting model in Ref. [2]. Hence, we see again the asymptotical conformal invariance of the model (1) in the infrared asymptotic regime.

Summing up, we have considered in this work the model of Ref. [2], not just in the IR stable fixed point (as in [2]), but in the most general asymptotic regime. The theory under consideration possesses some unusual properties. For instance, there are in the theory some asymptotic regimes which are free of the zero-charge problem, either in the UV or in the IR limit. We have then considered the RG equations for the Newtonian and for the cosmological constants, and found that in both the UV and IR regions the theory may exhibit asymptotic regimes with decreasing cosmological constant and (or) increasing Newton running coupling. An estimation of the leading-log corrections to the static gravitational potential has been also given. It would be of interest to construct the supersymmetric version of the theory (1) (for a general introduction to supersymmetric theories, see [15]) which should describe the superconformal anomaly induced action. Then, using a technique similar to the one above we would be able to provide a description of quantum supergravity at large distances.

\section{Acknowledgments}

The authors thank R. Tarrach for helpful remarks. SDO and ILS would like to acknowledge the kind hospitality of the members of the Dept. ECM, Barcelona University and of the Particle Physics Group, Hiroshima University, respectively. This work has been supported in part by DGICYT (Spain), project no. PB90-0022, by CIRIT (Generalitat de Catalunya), and by the Russian Foundation for Fundamental Research, project no. 94-02-03234. 


\section{References}

[1] Weinberg S., Rev. Mod. Phys. 61 (1989) 1.

[2] Antoniadis I., Mottola E., Phys. Rev. D45 (1992) 2013.

[3] Antoniadis I., Mazur P.O., Mottola E., Nucl. Phys. B388 (1992) 627;

Odintsov S.D., Z. Phys. C54 (1992) 531.

[4] Deser S., Duff M.J., Isham C., Nucl. Phys.111B (1976) 45;

Birrell N.D., Davies P.C.W., Quantum Fields in Curved Space-Time (Cambridge University Press, 1982).

[5] Tsamis N.C., Woodard R.P. Preprint UFIFT (1994).

[6] Elizalde E., Jacksenaev A.G., Odintsov S.D., Shapiro I.L. Preprint UB-ECM-94/5 (1994), Phys. Lett. B, to appear.

[7] Reigert R.Y., Phys. Lett. 134B (1984) 56;

Fradkin E.S., Tseytlin A.A., Phys. Lett.134B (1984) 187;

Buchbinder S.D., Odintsov S.D., Shapiro I.L., Phys. Lett.162B (1985) 93.

[8] Tomboulis E.T., Nucl. Phys. 329B (1990) 410;

Odintsov S.D., Shapiro I.L., Class.Quant.Grav. 8 (1991) L57.

[9] Stelle K., Phys. Rev. D 16 (1977) 953.

[10] Buchbinder S.D., Odintsov S.D., Shapiro I.L., Effective Action in Quantum Gravity (IOP, Bristol, 1992).

[11] Odintsov S.D., Pérez-Mercader J., Int. J. Mod. Phys. D1 (1993) 401.

[12] Goldman T., Pérez-Mercader J., Cooper F., Martin-Nieto M., Phys. Lett. 281B (1992) 219.

[13] Donoghue J.F. Preprint UMHEP-396 (1993) 401.

[14] Taylor T.R. and Veneziano G., Nucl. Phys. B 345 (1990) 210.

[15] West P., Introduction to Supersymmetry and Supergravity (World Scientific, Singapore, 1986). 